\title{
Status of the XENON100 experiment for WIMP direct detection
}

\author{
Alfredo D. Ferella*† \\ Physics Institute, University of Zürich, Winterthurerstrasse 190, CH-8057, Zürich, Switzerland \\ E-mail: ferella@physik.uzh.ch
}

The XENON100 experiment aims to directly detect cold dark matter particles via their collisions with xenon nuclei. For this purpose an ultra-low background double phase (liquid-gas) xenon filled time projection chamber with a total mass of $170 \mathrm{~kg}$ ( $\sim 65$ in the target region and $\sim 105$ in the active shield) has been installed at the Gran Sasso Underground Laboratory and is currently taking data. In this paper the background predictions based on Monte Carlo simulations with input from screening of detector and shield materials are presented together with the current status of the experiment.

European Physical Society Europhysics Conference on High Energy Physics, EPS-HEP 2009, July 16 - 222009

Krakow, Poland

\footnotetext{
* Speaker.

†n behalf of the XENON100 Collaboration
} 


\section{Introduction}

An increasing number of astrophysical and astronomical observations points to the existence of a non luminous, non baryonic and cold (i.e. non relativistic) matter component of the Universe, therefore called Cold Dark Matter (CDM) [1, 2,3]. The Dark Matter particles are still undetected, but they have to belong to a category beyond the Standard Model; the most appealing and interesting candidates are the so called Weakly Interactive Massive Particles (WIMPs) predicted by Supersymmetric theories (SUSY), models with extra dimensions and little Higgs models $[4,5,6,7]$. Different WIMP detection approaches are used in order to determine the characteristics of such particles: experiments at LHC will try to look for their appearance in the collisions; high energy astro-particle physics experiments are looking for WIMP annihilation signatures from the center of the Sun or from the center of the Galaxy and Dark Matter direct detection experiments are looking for the elastic scattering of WIMPs off the target nuclei.

Among the latter category, the XENON project is one of the most promising: after the successful results of a first $10 \mathrm{~kg}$ scale detector, XENON10 [8, 9], the XENON collaboration designed and built a second generation experiment exploiting the same "double-phase" (liquid-gas) time projection chamber (TPC) technique based on xenon, with a mass increase by a factor 10 and a radioactive background level reduction of a factor 100. The aim of the experiment, XENON100, is to reach a sensitivity of a factor $\sim 50$ better than XENON10.

\section{The XENON100 experiment}

The use of liquid xenon as target for a Dark Matter direct search experiment is motivated by several reasons: it has high density $(\rho \sim 3 \mathrm{~g} / \mathrm{cm})$ allowing ton scale detectors of relatively small sizes; has a high atomic number $(\mathrm{A} \sim 131)$ which enhances the probability of WIMP-nucleus interactions $\left(\sigma \propto A^{2}\right)$; natural xenon has $\sim 50 \%$ abundance of odd isotopes, which allows to probe also the WIMP spin dependent interactions; a low energy threshold is achievable thanks to the high ionization and scintillation yield in Liquid Xenon. Moreover the requirement of low background is fulfillable, given the absence of any long lived radioactive isotope in the noble liquid and considering its efficiency as self-shielding target, given the high Z (54). The latter feature, combined with the possibility of reconstructing the position of the interactions in 3D allows background reduction by volume fiducialization.

The XENON100 detector is a dual-phase (liquid-gas) Time Projection Chamber (TPC) filled with Xenon ( $\sim 65 \mathrm{~kg}$ of target volume) surrounded by $\sim 105 \mathrm{~kg}$ of liquid xenon acting as a veto. The TPC is enclosed in a PTFE (Teflon) cylinder of $30 \mathrm{~cm}$ height and $\sim 30 \mathrm{~cm}$ diameter (Teflon is a suitable plastic with very good UV light reflective features [10]). The whole volume is viewed by a total of 242 squared (1" $\times 1$ ") low radioactivity photo-multiplier tubes (Hamamatsu R8520-06$\mathrm{Al}$ ), especially developed to be operational at the Liquid Xenon temperature for the characteristic xenon scintillation wavelength $(\lambda=178 \mathrm{~nm}) .98$ of these PMTs are on the top of the TPC in the gas phase, arranged in concentric circles to improve position reconstruction, whereas 80 PMTs are on the bottom of the TPC placed very close to each other in order to optimize the light collection. The remaining 64 PMTs are placed in the veto, looking at the side, at the top and at bottom around the TPC. A detailed description of the XENON100 detector is shown in Fig. 1. 


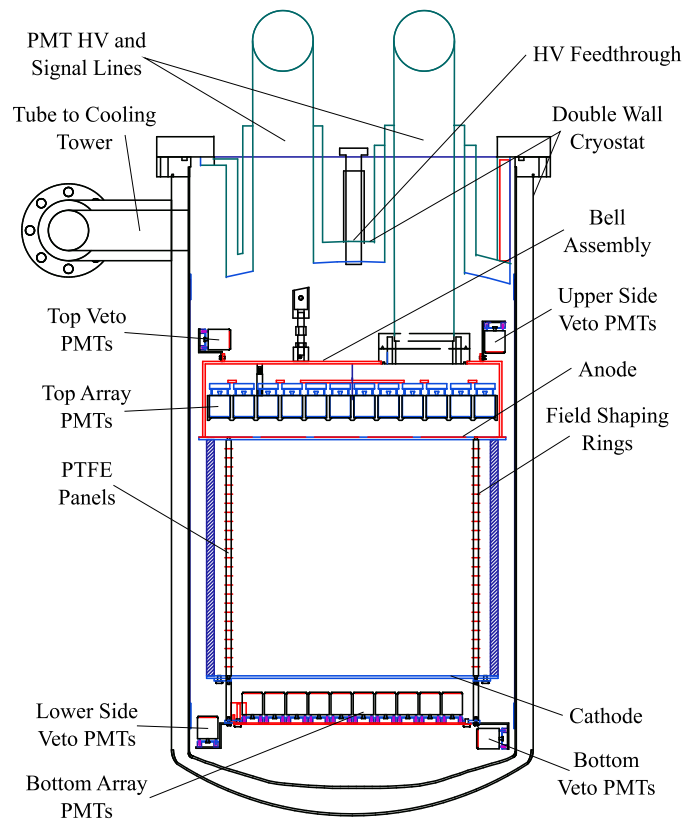

Figure 1: Schematic view of the XENON100 detector. The detector is fully assembled and installed underground at LNGS since February 2008. The TPC is enclosed in a PTFE cylinder, made with 24 interlocking panels which support the field shaping wires. The TPC volume is closed at the bottom by a cathode mesh, and at the top by 3 meshes, a central anode sandwiched by two grounded meshes. Custom-made, low radioactivity, high voltage feedthroughs are used to bias cathode and anode. The materials used for the detector were carefully selected for having low radioactivity content and the detector design is such that all radioactive "hot" components are located far away from the sensitive volume, outside the shield.

The cryostat is a double wall, vacuum insulated, stainless steel vessel where the Xenon is kept liquid using a Pulse Tube Refrigerator (PTR) which shows stable performances over long operating time. A high temperature getter is used in order to continuously purify the Xenon from electronegative impurities which attenuate ionization signals due to electron absorption. Gain measurements of the PMTs are performed on a regular basis in order to monitor the stability and performance of the PMTs. A slow control system monitors all important parameters.

The basic working principles of XENON100 are the following: a particle interacting in the Liquid Xenon target generates a prompt scintillation signal (S1) which is detected by the two arrays of PMTs. The ionization electrons produced in the same interaction are drifted towards the anode, in an electric field across the TPC (of the order of $\sim 1 \mathrm{kV} / \mathrm{cm}$ ), and once they reach the liquid-gas interface, given the high $(\sim 13 \mathrm{kV} / \mathrm{cm})$ field in the $2.5 \mathrm{~mm}$ gas gap between the first grid and the anode (see Fig. 1 for details) such electrons are extracted from the liquid and accelerated in the gas, producing a secondary scintillation signal (S2), proportional to the ionization charge, which is also detected by the two arrays of PMTs. The time distance between S1 and S2 (drift time), since the electron drift velocity in liquid xenon is constant $(\sim 2 \mathrm{~mm} / \mu \mathrm{s}$ at $1 \mathrm{kV} / \mathrm{cm})$, gives the information of the position of the interaction vertex in the vertical axis. Given that the diffusion of electrons in the liquid is very small and the S2 signal is very localized, the hit pattern of the secondary scintillation signal in the top PMT array can be used in order to reconstruct the interaction vertex in the horizontal plane. Three different methods are used to reconstruct the XY position, based on $\chi^{2}$ minimization, neural network and support vector machine algorithms. In this way a 3-dimensional event position information is available. Moreover the nature of the impinging particles can be discriminated by studying the S1 and S2 signals; for electron recoils (usual background in the energy region of interest for Dark Matter search) the ratio of S2/S1 is much higher than for nuclear recoils (expected WIMP signal), because of the different ionization density of the two particles.

The two-phase operation mode with S1 and S2 signals was started during Spring 2009. Purity 
Table 1: Results from some of the materials screened for radioactivity with high purity germanium spectrometers.

\begin{tabular}{|l|c|c|c|c|c|}
\hline Sample & Unit & ${ }^{232} \mathbf{T h}$ & ${ }^{238} \mathbf{U}$ & ${ }^{40} \mathbf{K}$ & ${ }^{60} \mathbf{C o}$ \\
\hline Innermost $\mathrm{Pb}($ shield$)$ & {$[\mathrm{mBq} / \mathrm{kg}]$} & $<0.55$ & $<0.66$ & $<1.46$ & $<0.11$ \\
\hline Outermost $\mathrm{Pb}($ shield$)$ & {$[\mathrm{mBq} / \mathrm{kg}]$} & $<0.43$ & $<0.92$ & $<14 \pm 36$ & $<0.12$ \\
\hline Polyethylene $($ shield $)$ & {$[\mathrm{mBq} / \mathrm{kg}]$} & $<0.094$ & $0.23 \pm 0.05$ & $0.7 \pm 0.4$ & N.A. \\
\hline Stainless Steel & {$[\mathrm{mBq} / \mathrm{kg}]$} & $<1.9$ & $<1$ & $8.5 \pm 0.9$ & $10.5 \pm 4.2$ \\
\hline PTFE & {$[\mathrm{mBq} / \mathrm{kg}]$} & $<0.16$ & $<0.31$ & $<2.25$ & $<0.11$ \\
\hline 22 QE PMTs & {$[\mathrm{mBq} / \mathrm{PMT}]$} & $0.18 \pm 0.06$ & $<0.24$ & $11 \pm 2$ & $0.6 \pm 0.1$ \\
\hline
\end{tabular}

is still increasing and the maximum electron lifetime has not yet been achieved. Events with drift time of $160 \mu \mathrm{s}$, corresponding to the full length of the TPC are recorded. Gamma calibration runs are acquired on a daily basis in order to test the detector performances and study its features.

\section{Background}

To reduce the radioactivity from the detector materials detector and its shield and eventually improve the sensitivity of the experiment, a careful selection of all the materials has been done. This was performed based on the results of the radioactivity screening of many samples, mainly performed with High Purity Germanium spectrometers. The radioactive contaminations in a selection of materials used for the experiment is shown in table 1. Furthermore all the cryogenics and the electrical feedthroughs have been moved outside the shield in order to minimize the impact of all known sources of background. The radioactivity of all the components has been input in detailed Monte-Carlo simulations of the $\gamma$ and neutron background of the experiment. The expected rate of single interaction electron recoil signals is $24.05 \pm 0.17 \times 10^{-3}$ events $/ \mathrm{kg} / \mathrm{day} / \mathrm{keV}$ and 9.07 $\pm 0.14 \times 10^{-3}$ events $/ \mathrm{kg} / \mathrm{day} / \mathrm{keV}$ for 50 and $30 \mathrm{~kg}$ fiducial mass respectively; whereas the total neutron induced single nuclear recoil rates are $1.62 \mathrm{n} /$ year and $0.60 \mathrm{n} / \mathrm{year}$ for 50 and $30 \mathrm{~kg}$ fiducial mass respectively.

A first WIMP search will be performed with the $50 \mathrm{~kg}$ target, which is expected to be background free for 40 days, leading to a spin-independent WIMP-nucleon interaction cross section sensitivity of $\sigma \sim 6 \times 10^{-45} \mathrm{~cm}^{2}$ for $100 \mathrm{GeV} / \mathrm{c}^{2}$ WIMP mass. This limit improves to $\sigma \sim 2 \times 10^{-45}$ $\mathrm{cm}^{2}$ after 200 days of data taking with $30 \mathrm{~kg}$ fiducial volume.

\section{References}

[1] W. Freedman and M. Turner, Rev. Mod. Phys., 75, 1433 (2003).

[2] D. Clowe et al., ApJ, 648, L109 (2006).

[3] M.J. Jee et al., http://xxx.lanl.gov/abs/0705.2171

[4] A. Bottino et al., Phys. Rev.D69 037302 (2004);

[5] J. Ellis et al., Phys. Rev.D71 095007 (2005);

[6] H. C. Cheng et al., Phys. Rev. Lett. 89211301 (2002);

[7] A. Birkedal-Hansen and J. G. Wacker, Phys. Rev.G69 065022 (2004).

[8] J. Angle et al. (XENON10 Collaboration), Phys. Rev. Lett. 100, 021303 (2008).

[9] J. Angle et al. (XENON10 Collaboration), Phys. Rev. Lett. 101 (2008) 091301.

[10] M. Yamashita et al., NIM A 535, 692 (2004). 